\title{
Chiral Anion-Mediated Asymmetric Ring Opening of meso-Aziridinium and Episulfonium lons
}

\author{
Gregory L. Hamilton, Toshio Kanai, and F. Dean Toste* \\ Department of Chemistry, University of California, Berkeley, California 94720
}

Received August 13, 2008; E-mail: fdtoste@ berkeley.edu

Recently, chiral anions have been uncovered as a potent means for inducing asymmetry in catalytic reactions. ${ }^{1}$ Introduced in the arena of cationic transition metal catalysis in 2000 by Arndtsen and co-workers, ${ }^{2}$ the strategy has recently been demonstrated by our group and others to be capable of providing products with exceptional enantioselectivity, even in cases where chiral ligands have failed. ${ }^{3,4}$ Parallel to these discoveries, work spearheaded by List has successfully utilized chiral counteranions as supplements or alternatives to chiral amines in iminium-based organocatalytic reactions. ${ }^{5}$ Instrumental to all these successes has been the use of axially chiral phosphate ions initially developed by Akiyama and Terada as chiral Brønsted acids for enantioselective additions to imines. ${ }^{6-8}$ A goal of our laboratory has been probing the stereodifferentiating ability of these chiral phosphates beyond cases involving a putative hydrogen bond to an imine or other electrophile. Moving forward from the existing precedent with an eye toward transformations without a current asymmetric variant, we reasoned that chiral anions could provide a much-needed solution to the problem of reactions proceeding through cationic intermediates that do not possess a (Lewis or Brønsted) basic site capable of coordinating to a metal or proton. One approach to this problem, advanced quite recently by Jacobsen and colleagues, involves the binding of a halide counteranion by a chiral thiourea catalyst, thereby enabling asymmetric additions to iminium and oxocarbenium intermediates. ${ }^{9,10}$

We envisioned a complementary strategy initiated by an "anion metathesis," wherein the silver salt of a chiral anion could abstract a halide from an organic fragment to produce a chiral ion pair (eq 1). The resulting cationic intermediate could then be intercepted by a nucleophile in an enantioselective manner directed by the chiral counteranion. After proton transfer, this sequence would result in the desired optically active substitution product and the conjugate acid of the chiral anion.

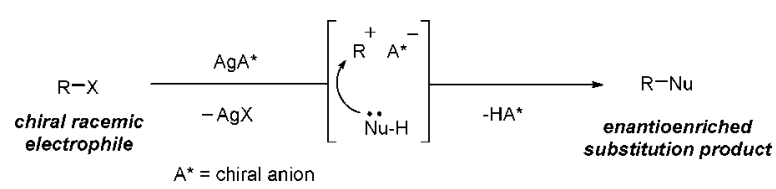

The challenge for this seemingly intuitive approach results from reforming the chiral silver salt so that the overall transformation is catalytic in the chiral material. Addition of an achiral $\mathrm{Ag}(\mathrm{I})$ source to the reaction mixture in order to turn over the chiral anion necessarily brings an accompanying achiral counteranion that can lead to racemic product. An attractive solution came to us in the form of establishing a phase separation between the substrate and chiral anion catalyst (bulk solution) and the stoichiometric silver salt (solid phase). The resulting catalytic cycle can be thought of as chiral anion phase transfer catalysis in analogy to the established chiral cation variety (Figure 1). ${ }^{11-13}$

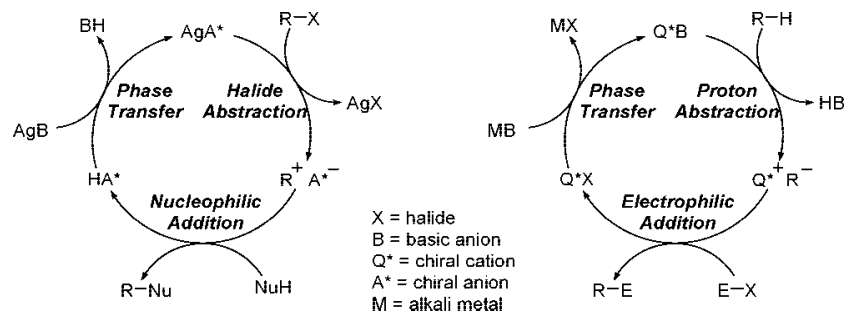

Figure 1. Proposed chiral anion phase transfer catalysis (left) as compared to chiral cation PTC.

Table 1. Reaction Development

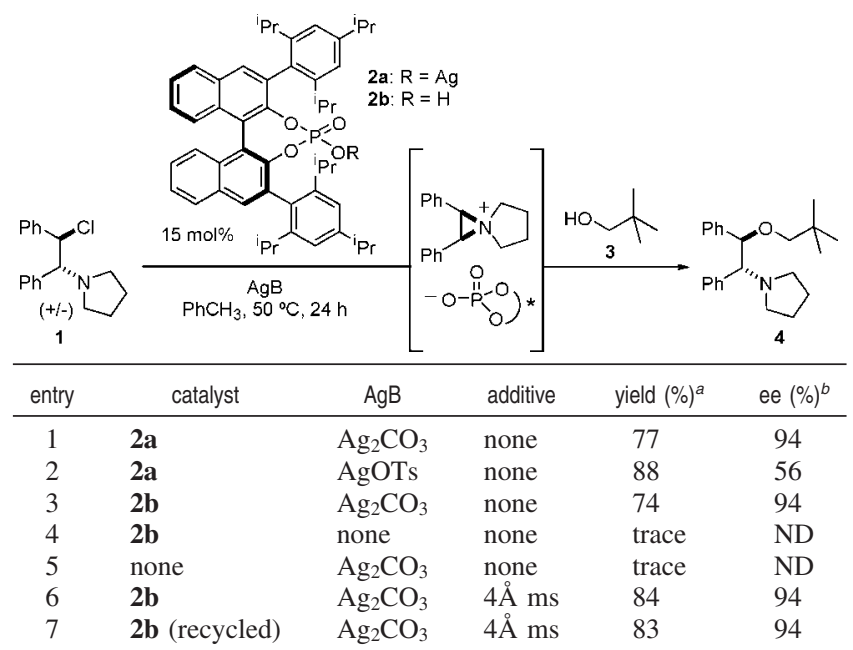

${ }^{a}$ Conditions: $15 \mathrm{~mol} \%$ 2, 1.2 equiv (in $\mathrm{Ag}$ ) $\mathrm{AgB}, 4$ equiv 3, $0.1 \mathrm{M}$ in toluene, $50{ }^{\circ} \mathrm{C}, 24 \mathrm{~h}$. Yields refer to isolated material. ${ }^{b}$ Determined by ${ }^{1} \mathrm{H}$ NMR using chiral shift reagent; see Supporting Information.

Surprisingly, examination of the literature reveals no successful examples of chiral anion phase transfer catalysis, although we came across one report of work aiming toward the concept. ${ }^{14}$ In it the authors investigated the asymmetric ring opening of meso-aziridinium ions generated from ring closure of $\beta$-chloro tertiary amines, obtaining products with a maximum of $30 \%$ yield and $15 \%$ ee. No attempt was made to achieve catalytic turnover. This transformation sparked our interest for two reasons. First, since the meso intermediate is a quaternary-substituted aziridinium ion it is unable to be activated by a Brønsted or Lewis acid. Furthermore, the reaction generates two stereocenters and allows a direct synthesis of valuable unprotected amines. ${ }^{15-17}$

We initiated our investigation with racemic chloroamine $\mathbf{1}$ and neopentyl alcohol as nucleophile (Table 1). For chiral silver salt we chose the binaphthol-phosphate $\mathbf{2}$ that has proven successful for chiral counteranion-based reactions developed by our group and others. ${ }^{3,5}$ Our confidence in this anion was further bolstered by the 
Scheme 1. Nucleophile and Substrate Scope ${ }^{a}$
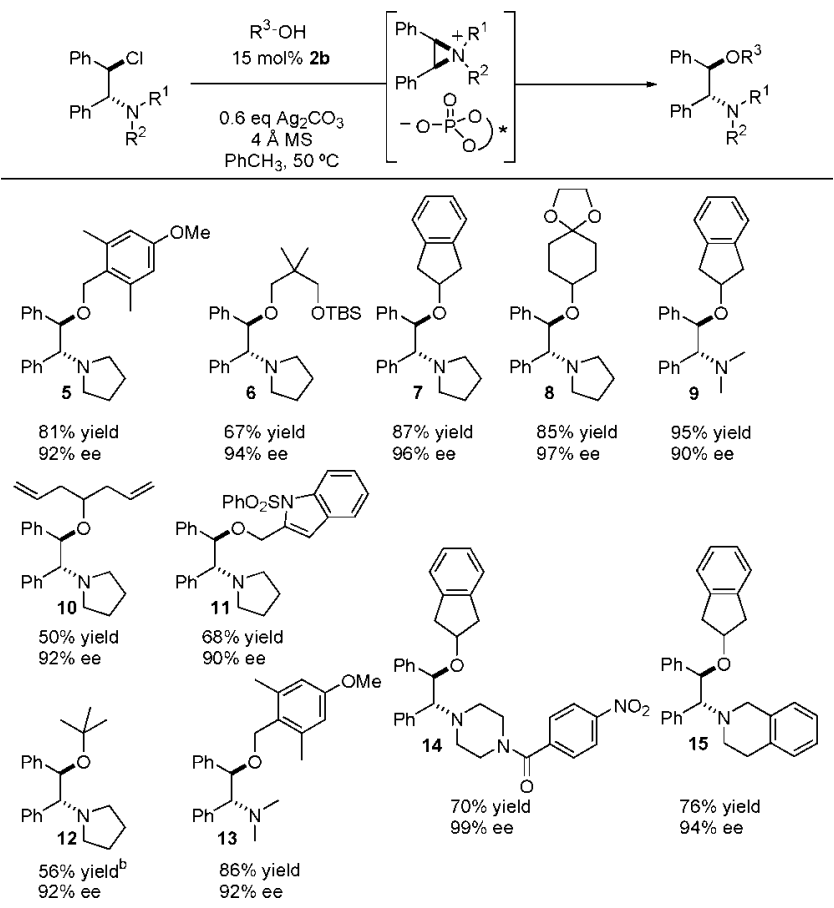

${ }^{a}$ Conditions: 15 mol \% $2 \mathbf{b}, 0.6$ equiv $\mathrm{Ag}_{2} \mathrm{CO}_{3}, 4$ equiv $\mathrm{R}^{3} \mathrm{OH}, 0.1 \mathrm{M}$ in toluene, $4 \AA \mathrm{MS}, 50{ }^{\circ} \mathrm{C}, 24-36 \mathrm{~h}$. Yields refer to isolated material; ee's determined by chiral HPLC or ${ }^{1} \mathrm{H}$ NMR using chiral shift reagent, see Supporting Information. ${ }^{b}$ Run using 30 equiv ${ }^{t} \mathrm{BuOH}$.

high solubility of its salts in organic solvents, a property that facilitates the phase transfer process.

As mentioned previously, we expected that the choice of stoichiometric silver salt would prove critical. Testing several insoluble basic silver compounds revealed that $\mathrm{Ag}_{2} \mathrm{CO}_{3}$ efficiently turned over the catalyst while being mild enough to not significantly react with starting materials or products: using $15 \mathrm{~mol} \%$ of the silver phosphate salt $\mathbf{2 a}$ and a slight excess of $\mathrm{Ag}_{2} \mathrm{CO}_{3}$ we obtained the desired substitution product in good yield and excellent ee (Table 1, entry 1). In contrast, and as expected, addition of more soluble silver compounds such as AgOTs led to significantly lower ee due to competition from the achiral anion (entry 2). Only the shown diastereomer resulting from double inversion was obtained; in no cases did we observe competition from a direct $\mathrm{S}_{\mathrm{N}} 2$ substitution of the chloride.

Importantly, since $\mathrm{Ag}_{2} \mathrm{CO}_{3}$ rapidly generates the silver phosphate salt from the corresponding phosphoric acid, the more easily prepared and stored $\mathbf{2 b}$ performs equally well as precatalyst (entry 3). Accordingly, control experiments demonstrated that both the chiral phosphoric acid and $\mathrm{Ag}_{2} \mathrm{CO}_{3}$ are necessary for the reaction to proceed (entries 4 and 5). The failure of $\mathrm{Ag}_{2} \mathrm{CO}_{3}$ to promote the reaction by itself is consistent with the phase transfer role of the phosphate anion; without it, there is no $\mathrm{Ag}^{+}$in solution. By way of yield optimization, it was found to be desirable to add powdered $4 \AA$ molecular sieves to minimize competitive nucleophilic addition of the $\mathrm{H}_{2} \mathrm{O}$ equivalent generated by protonation of $\mathrm{Ag}_{2} \mathrm{CO}_{3}$ (entry 6 ). Finally, we wanted to establish that the catalyst could be recycled. Simple reacidification by aqueous workup of the phosphate following normal chromatographic purification allows recovery of $\mathbf{2 b}$ in quantitative yield. Catalyst obtained thusly provides product with identical yield and ee when used in another reaction (entry 7).

Next we turned to establishing the scope of the enantioselective ring opening by varying the alcohol nucleophile (Scheme 1).
Secondary, tertiary, and relatively hindered primary alcohols all react with high enantioselectivity. Notably, these classes of alcohols generate products that would be generally difficult to prepare by a traditional Williamson ether synthesis due to competing elimination and sluggish reactivity of the necessary electrophiles. In addition to structural variation, functional group tolerance was also found to be high, with acid-labile (acetal, silyl ether) and oxidationsensitive (indole, electron rich benzyl alcohol) functionalities surviving the reaction conditions without incident.

Our reaction can also be applied to substrates containing different amine substituents. Acyclic (dimethyl) substitution, other ring sizes, heteroatoms, and fused rings are well tolerated. Furthermore, tetrahydroisoquinoline $\mathbf{1 5}$ demonstrates that the amine need not be symmetrically substituted $\left(\mathrm{R}^{1} \neq \mathrm{R}^{2}\right)$.

We also investigated a chiral racemic alcohol as nucleophile in an effort to obtain a product with three stereocenters. Since the alcohol is used in excess relative to the more expensive amine fragment, $100 \%$ theroretical yield of one diastereomer of the desired adduct is possible assuming the catalyst can mediate an efficient kinetic resolution. In fact, when the reaction was performed using racemic alcohol $\mathbf{1 6}$ it proceeded as we hoped to furnish product $\mathbf{1 7}$ with exceptional de and ee (eq 2).

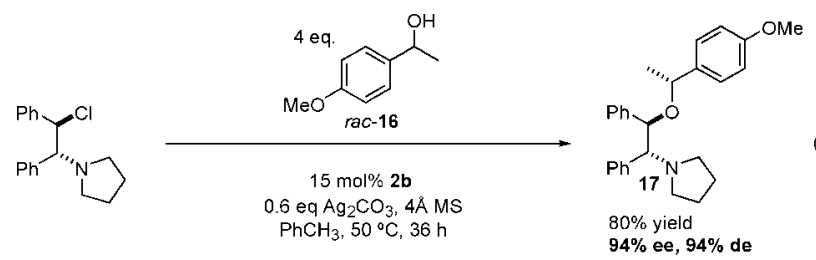

Although a valuable feature of the described reaction is the direct synthesis of substituted $\beta$-alkoxy amines, 1,2-amino alcohols can also be prepared by a two-step procedure. We found that 2,6dimethyl-4-methoxybenzyl ether $\mathbf{5}$ could be efficiently cleaved by the agency of CAN without significant overoxidation (eq 3 ).<smiles>COc1cc(C)c(COC(c2ccccc2)C(c2ccccc2)N2CCCC2)c(C)c1</smiles>
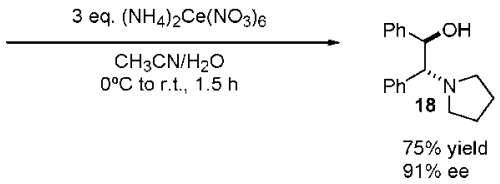

During studies on the aziridinium opening reaction, we hypothesized that our chiral anion strategy could be applied to a wide variety of cationic reactions, as long as we could selectively generate the key cationic intermediate as an ion pair with a chiral counteranion. We considered a reasonable extension of our chemistry to the sulfur analogue of the ring opening reaction: namely, the desymmetrization of meso-episulfonium ions, ${ }^{18}$ however, it was apparent that we could not simply employ our silver-halide abstraction method of cation generation, since the sulfide would likely bind $\mathrm{Ag}^{+}$. Instead, we settled on trichloroacetimidate as a leaving group that could be directly activated by a chiral phosphoric acid (Scheme 2). After protonation of the imidate, loss of trichloroacetamide and ring closure would generate the desired episulfonium ion paired with the chiral phosphate. Interception of the intermediate with a nucleophile would furnish the chiral sulfide product and complete the catalytic cycle after subsequent proton transfer.

In practice, we were pleased to find that the reaction worked as designed. A small sample of substrates containing different sulfur substituents reacted with various alcohols to provide in all cases 
Scheme 2. Asymmetric Opening of Episulfonium Ions ${ }^{a}$
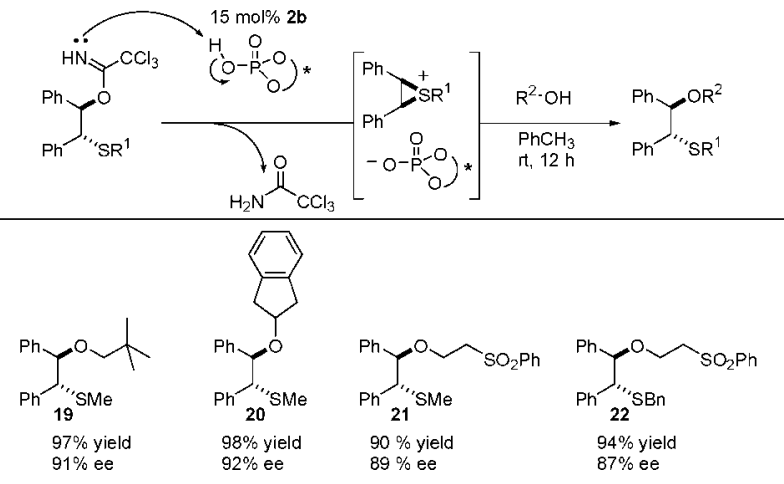

${ }^{a}$ Conditions: $15 \mathrm{~mol} \% \mathbf{2 b}, 2$ equiv $\mathrm{R}^{2} \mathrm{OH}, 0.1 \mathrm{M}$ in toluene, room temp, $12 \mathrm{~h}$. Yields refer to isolated material; ee's determined by chiral HPLC, see Supporting Information.

products in very high yield and good ee (Scheme 2). Although the reaction is initiated by protonation by the phosphoric acid, it is clearly mechanistically distinct from chiral Brønsted catalyzed reactions where a prochiral imine is activated by an acid. Since the trichloroacetimidate starting material is chiral while the intermediate episulfonium is meso, the ring opening must be enantiodetermining. Therefore the enantioselectivity likely results from an ion pair with the chiral anion rather than a hydrogen bond to the electrophile.

In summary, we have developed two enantioselective ring opening reactions of cationic intermediates that could not be readily achieved using previously established methods of asymmetric catalysis. In doing so we have expanded the range of chiral counteranion-mediated reactions beyond iminium and oxocarbenium based transformations. Furthermore, we have demonstrated that a primary barrier to designing enantioselective reactions of more types of cationic intermediates is the selective generation of the critical chiral ion pair. Toward that end, we have shown here the success of two methods: one based on a unique chiral anion phase transfer catalytic cycle and the other on activation of trichloroacetimidates. The flexibility of the two strategies should facilitate new reaction development since either an organohalide or an alcohol (in the form of the imidate) can serve as precursor.

Acknowledgment. We gratefully acknowledge Merck Research Laboratories, Bristol-Myers Squibb, and Novartis for funding. G.L.H. thanks the NSF for a graduate fellowship. T.K. thanks Dainippon Sumitomo Pharma for funding and research opportunity.

Supporting Information Available: Experimental procedures and compound characterization data. This material is available free of charge via the Internet at http://pubs.acs.org.

\section{References}

(1) For a review on chiral anions, see: Lacour, J.; Hebbe-Viton, V. Chem. Soc. Rev. 2003, 32, 373.
(2) (a) Llewellyn, D. B.; Adamson, D.; Arndtsen, B. A. Org. Lett. 2000, 2, 4165. For follow-up research, see:(b) Llewellyn, D. B.; Arndtsen, B. A. Tetrahedron: Asymmetry 2005, 16, 1789. Another study: (c) Dorta, R.; Shimon, L.; Milstein, D. J. Organomet. Chem. 2004, 689, 751.

(3) (a) Hamilton, G. L.; Kang, E. J.; Mba, M.; Toste, F. D. Science 2007, 317, 496. (b) Mukherjee, S.; List, B. J. Am. Chem. Soc. 2007, 129, 11336.

(4) For examples of chiral Brønsted acids used in cooperation with transition metals, see:(a) Komanduri, V.; Krische, M. J. J. Am. Chem. Soc. 2006, 128, 16448. (b) Rueping, M.; Antonchick, A. P.; Brinkmann, C. Angew. Chem., Int. Ed. 2007, 46, 6903.

(5) (a) Mayer, S.; List, B. Angew. Chem., Int. Ed. 2006, 45, 4193. (b) Martin, N. J. A.; List, B. J. Am. Chem. Soc. 2006, 128, 13368. (c) Wang, X.; List, B. Angew. Chem., Int. Ed. 2008, 47, 1119. (d) Wanner, M. J.; van der Haas, R. N. S.; de Cuba, K. R.; van Maarseveen, J. H.; Hiemstra, H. Angew. Chem., Int. Ed. 2007, 46, 7485. For a related study using Brønsted acid catalysis, see:(e) Storer, R. I.; Carrera, D. E.; Ni, Y.; MacMillan, D. W. C. J. Am. Chem. Soc. 2006, $128,84$.

(6) (a) Uraguchi, D.; Terada, M. J. Am. Chem. Soc. 2004, 126, 5356. (b) Akiyama, T.; Itoh, J.; Yokota, K.; Fuchibe, K. Angew. Chem., Int. Ed. 2004, $43,1566$.

(7) As early as ref $6 \mathrm{~b}$ the limiting case transition state of fully ionized phosphate/ iminium ion pair was proposed, thereby foreshadowing the future developments in chiral counteranion chemistry.

(8) Reviews on chiral Brønsted acids: (a) Akiyama, T. Chem. Rev. 2007, 107, 5744. (b) Akiyama, T.; Itoh, J.; Fuchibe, K. Adv. Synth. Catal. 2006, 348, 999. (c) Connon, S. J. Angew. Chem., Int. Ed. 2006, 45, 3909.

(9) (a) Raheem, I. T.; Thiara, P. V.; Peterson, E. A.; Jacobsen, E. N. J. Am. Chem. Soc. 2007, 129, 13404. (b) Raheem, I. T.; Thiara, P. V.; Jacobsen, E. N. Org. Lett. 2008, 10, 1577. (c) Reisman, S. E.; Doyle, A. G.; Jacobsen, E. N. J. Am. Chem. Soc. 2008, 130, 7198.

(10) For use of a stoichiometric chiral anion in an asymmetric Stevens rearrangement: Gonçalves-Farbos, M-H.; Vial, L.; Lacour, J. Chem. Commun. 2008, 829.

(11) Reviews on chiral phase transfer catalysis: (a) Ooi, T.; Maruoka, K. Angew. Chem., Int. Ed. 2007, 46, 4222. (b) O'Donnell, M. J. In Catalytic Asymmetric Synthesis, 2nd ed.; Ojima, I., Ed.; Wiley-VCH: New York, 2000; Chapter 10, pp 727-755. (c) O'Donnell, M. J. Acc. Chem. Res. 2004, 37, 506. (d) Lygo, B.; Andrews, B. I. Acc. Chem. Res. 2004, 37, 518.

(12) For selected recent examples of chiral cation phase transfer catalysis, see: (a) Elsner, P.; Bernardi, L.; Salla, G. D.; Overgaard, J.; Jørgensen, K. A. J. Am. Chem. Soc. 2008, 130, 4897. (b) Moss, T. A.; Fenwick, D. R.; Dixon, D. J. J. Am. Chem. Soc. 2008, 130, 10076. (c) Wang, X.; Kitamura, M.; Maruoka, K. J. Am. Chem. Soc. 2007, 129, 1038. (d) Poulsen, T. B.; Bernardi, L.; Alemán, J.; Overgaard, J.; Jørgensen, K. A. J. Am. Chem. Soc. 2007, 129, 441 .

(13) Many chiral phase transfer reactions go by an interfacial mechanism; this is possible for our concept also but is not shown for simplicity.

(14) Carter, C.; Fletcher, S.; Nelson, A. Tetrahedron: Asymmetry 2003, 14, 1995.

(15) For selected studies of biologically active compounds bearing a trans- $\beta$ aminoether motif, see: (a) Plouvier, B.; Beatch, G. N.; Jung, G. L.; Zolotoy, A.; Sheng, T.; Clohs, L.; Barrett, T. D.; Fedida, D.; Wang, W. Q.; Zhu, J. J.; Liu, Y.; Abraham, S.; Lynn, L.; Dong, Y.; Wall, R. A.; Walker, M. J. A. J. Med. Chem. 2007, 50, 2818. (b) Rebollo, O.; del Olmo, E.; Ruiz, G.; Lopez-Perez, J. L.; Gimenez, A.; San Feliciano, A. Bioorg. Med. Chem. Lett. 2008, 18, 184. (c) Witty, D. R.; Bateson, J.; Hervieu, G. J.; Al-Barazanji, K.; Jeffrey, P.; Hamprecht, D.; Haynes, A.; Johnson, C. N.; Muir, A. I.; O'Hanlon, P. J.; Stemp, G.; Stevens, A. J.; Thewlis, K.; Winborn, K. Y. Bioorg. Med. Chem. Lett. 2006, 16, 4872. (d) Takeuchi, K.; Kohn, T. J.; Sall, D. J.; Denney, M. L.; McCowan, J. R.; Smith, G. F.; Gifford-Moore, D. S. Bioorg. Med. Chem. Lett. 1999, 9, 759.

(16) For asymmetric ring opening of protected aziridines with $\mathrm{TMSN}_{3}$, see:(a) Fukuta, Y.; Mita, T.; Fukuda, N.; Kanai, M.; Shibasaki, M. J. Am. Chem. Soc. 2006, 128, 6312. (b) Li, Z.; Fernández, M.; Jacobsen, E. N. Org. Lett. 1999, 1, 1611. With TMSCN: (c) Mita, T.; Fujimori, I.; Wada, R.; Wen, J.; Kanai, M.; Shibasaki, M. J. Am. Chem. Soc. 2005, 127, 11252.

(17) Recently a chiral phosphoric acid has also been used for desymmetrization of meso-aziridines with $\mathrm{TMSN}_{3}$. In this case covalent activation of benzoylated aziridine by silylated phosphate was proposed: Rowland, E. B.; Rowland, G. B.; Rivera-Otero, E.; Antilla, J. C. J. Am. Chem. Soc. 2007, 129, 12084.

(18) Episulfonium ions are intermediates in numerous reactions but have not been exploited in the context of asymmetric catalysis; for a review, see: Fox, D. J.; House, D.; Warren, S. Angew. Chem., Int. Ed. 2002, 41, 2462.

JA806431D 